\title{
Preliminary clinical study: percutaneous lumbar discectomy combined with external drainage for treatment of intervertebral disc infections with epidural abscess
}

\author{
Xiao Quanping ${ }^{1}$, Huanzhang Niu${ }^{1}$, Shuangying $\mathrm{Li}^{2}$ \\ ${ }^{1}$ Interventional Department, The First Affliated Hospital, and College of Clinical Medicine of Henan University of Science and Technology, \\ Luoyang, China \\ 2Urology Department, The LuoYang DongFang Hospital, Luoyang, China
}

Videosurgery Miniinv 2020; 15 (4): 625-631

DOI: https://doi.org/10.5114/wiitm.2020.94152

\begin{abstract}
Introduction: Percutaneous lumbar discectomy (PLD) combined with external drainage (ED) is a new technique for the treatment of intervertebral disc infection with epidural abscess.

Aim: To discuss the feasibility, safety and efficacy of PLD and ED for the treatment of intervertebral disc infections with epidural abscess.

Material and methods: We enrolled 12 patients who underwent intervertebral disc infections with epidural abscess. The clinical efficacy was evaluated by visual analog scale (VAS) and standard Macnab's evaluation. Postoperative computed tomography and magnetic resonance imaging were also used to evaluate the clinical efficacy.

Results: The technical success rate is $100 \%$. Preoperation mean VAS score was $8.18 \pm 0.98 ; 5.36 \pm 1.50$ postoperation 1 month; $3.36 \pm 2.24$ postoperation 6 months; $2.77 \pm 0.31$ postoperation 12 months. The comparison of preoperation and postoperation VAS showed a significant difference $(p<0.05)$. According to standard Macnab's evaluation, of all 12 cases, postoperation 12 months - excellent 4 cases, good 7 cases, poor 1 case. The efficacy rate was 91.6\%. No serious complications were recorded.

Conclusions: Percutaneous lumbar discectomy combined with external drainage may be a safe and efficacy method for the treatment of intervertebral disc infections with epidural abscess.
\end{abstract}

Key words: percutaneous lumbar discectomy, external drainage, intervertebral disc infections with epidural abscess.

\section{Introduction}

Percutaneous lumbar discectomy (PLD) is a minimally invasive procedure for the treatment of intervertebral disc protrusion. It has been extensively applied for the treatment of lumbar disk herniation. External drainage (ED) is used for the drainage of abscesses of various organs. In clinical practice, intervertebral disc infection with epidural abscess is considered as an indication for surgery. So, PLD combined with ED for the treatment of intervertebral disc infection with epidural abscess is rarely reported. As an attempt, we adopted combined miniinvasive treatment to treat successfully 12 cases of patient with intervertebral disc infection with epidural abscess.

\section{Aim}

The aim of this study was to discuss the feasibility, safety and efficacy of PLD and ED for the treat-

\section{Address for correspondence}

Xiao Quanping MD, Interventional Department, The First Affliated Hospital, and College of Clinical Medicine of Henan University of

Science and Technology, 24 JingHua Road, 471003 LuoYang City, HeNan, China, phone: 15036550795, e-mail: xqp1981@126.com 
ment of intervertebral disc infection with epidural abscess.

\section{Material and methods}

Hospital ethics board human ethics approval was obtained prior to the initiation of this trial. We collected 12 cases of patients with intervertebral disc infection with epidural abscess who underwent PLD combined with ED between June 2010 and June 2012 in our department (Table I): 6 male cases $(n=6), 6$ female cases $(n=6)$. The average age was $58.3 \pm 1.2$ years. The enrolled patients were required to meet the following conditions: 1) they all showed the corresponding clinical symptoms and signs; 2) the symptoms had not been relieved or aggravated after conservative therapy; 3) intervertebral disc infections with epidural abscess were suggested on computed tomography $(\mathrm{CT})$ and magnetic resonance imaging (MRI) preoperatively; 4) the range of abscess was no more than three vertebral bodies (Photo 1).

\section{Apparatus and equipment}

Bard Truguide Coaxial Biopsy Needle (Bard Peripheral Vascular, Inc USA), working cannulas (Dragon-Crown, Jinan, China) penultimate cannula (Dragon-Crown, Jinan, China), a marrow nucleus rongeur (Dragon-Crown); guide wire (COOK, American); external drainage catheters (COOK, American); DSA
(Axiom Artis dra, Simmens, Germany), 3.0 T MRI (GE HDxt-3T, GE American) 64 Row CT (Toshiba Aquilion).

\section{Index of efficacy}

The clinical efficacy was evaluated by the standard Macnab's evaluation [1] and visual analog scale (VAS) scores. The pain intensity is evaluated by VAS (0-10; 0 represent no pain; 10 represents the worst pain). Improvement in Macnab's evaluation standard was divided into four grade. Excellent: No pain, no restriction of mobility, return to normal work and level of activity. Good: Occasional non-radicular pain, relief of presenting symptoms, able to return to modified work. Fair: Some improved functional capacity, still handicapped and/or employed. Poor: Continued objective symptoms of root involvement, additional operative invention needed at index level irrespective of length of postoperative follow-up. Postoperation CT (Toshiba Aquilion, Japan) and MRI (GE HDxt-3T, GE, American) of vertebrae was examined.

\section{Procedure}

Informed consent for participation in this study was obtained from the patient. A lateral position was required during the course of the operation under the guidance of the Digital subtraction angiography (DSA, Axiom Artis dra, Simmens, Germany). The

Table I. Information of patients

\begin{tabular}{|c|c|c|c|c|}
\hline Number & Gender [male/female] & Age [years] & Treated intervertebral disc & Therapy method \\
\hline 1 & Male & 49 & L3-4 & $P L D+E D$ \\
\hline 2 & Female & 50 & $\lfloor 4-5$ & $P L D+E D$ \\
\hline 3 & Male & 65 & $\lfloor 4-5$ & $P L D+E D$ \\
\hline 4 & Male & 60 & $\lfloor 4-5$ & $P L D+E D$ \\
\hline 5 & Female & 75 & L5-S1 & $P L D+E D$ \\
\hline 6 & Female & 68 & L3-4 & $P L D+E D$ \\
\hline 7 & Female & 72 & L4-5 & $P L D+E D$ \\
\hline 8 & Male & 47 & $\lfloor 4-5$ & $P L D+E D$ \\
\hline 9 & Female & 63 & L5-S1 & PLD + ED \\
\hline 10 & Male & 66 & $\lfloor 4-5$ & $P L D+E D$ \\
\hline 11 & Male & 54 & $\lfloor 4-5$ & $P L D+E D$ \\
\hline 12 & Female & 57 & L4-5 & PLD + ED \\
\hline
\end{tabular}



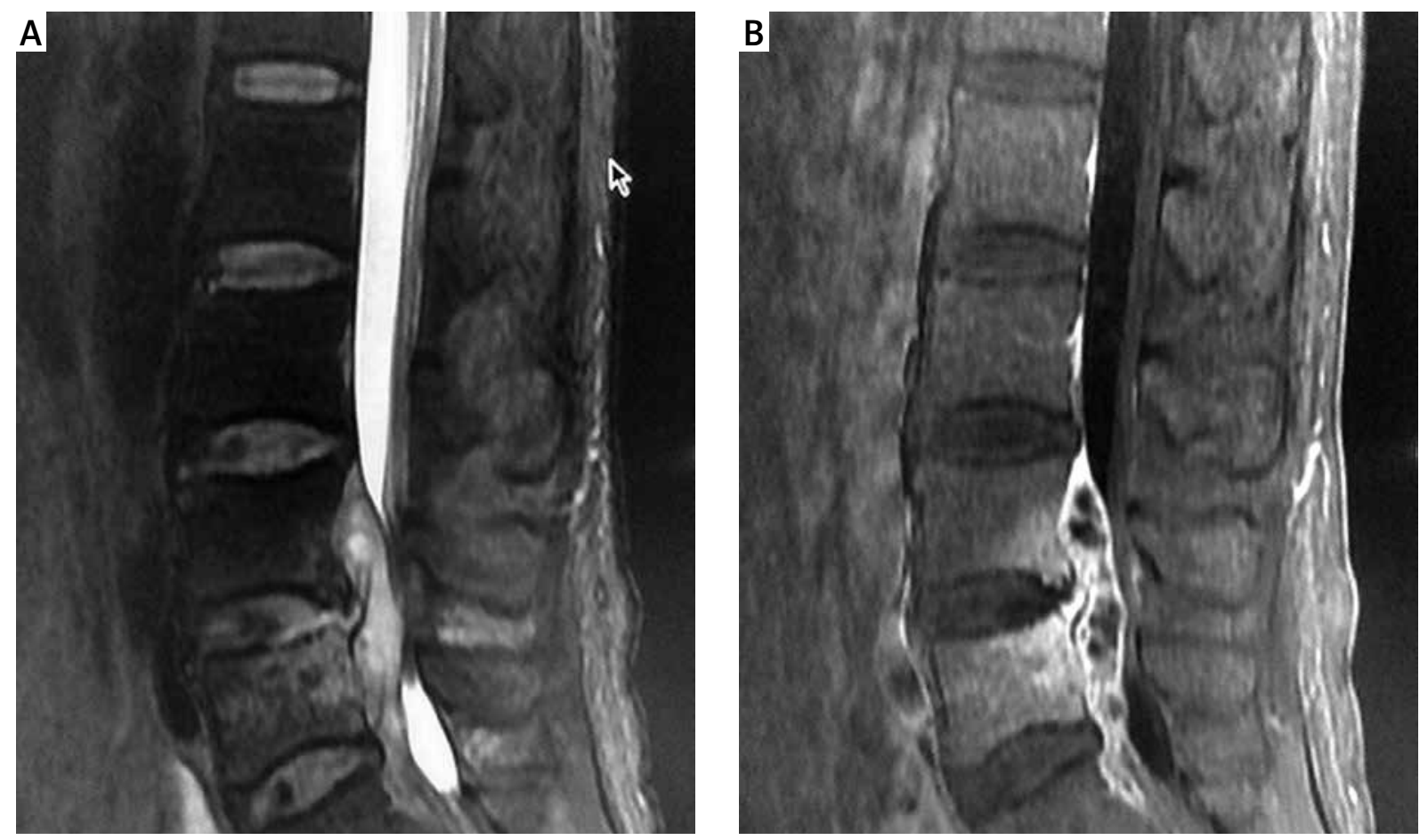

Photo 1. Non-enhanced Sagittal. A - T2-Weighted-MRI shows a fusiformis hyperintensity can be seen in the spinal dura involving the $L 5$ vertebral and the intervertebral disk at the L4-L5 levels. The spinal canal is stenosis. Enhanced sagittal. B - T1-weighted MRI demonstrates a fusiformis abnormal signal was enhancement inhomogeneous

patient's back skin was sterilized, and local anesthesia was applied on the puncture route. After administration of local anesthetic (2\% lidocaine), a small incision was made with a scalpel blade. With continuous fluoroscopic monitoring, a 15-G Bard Truguide Coaxial Biopsy Needle (Bard Peripheral Vascular, Inc USA) was inserted at the site of the anterior part of the L4-5 intervertebral disc, followed by sequential dilation of the tract with working cannulas (Dragon-Crown Co, Jinan, China) until the last working cannula (5 $\mathrm{mm}$ in diameter) reached the anterior portion of the L4-5 intervertebral disc. After removal of the penultimate cannula (Dragon-Crown, China), a marrow nucleus rongeur (Dragon-Crown, China) was inserted into the intervertebral disc through the last working cannula and the purulent materials and marrow tissues were extracted slowly, as much as possible (Photo 2). Then a 0.035 inch guide wire (COOK, American) was inserted through the work cannula (Photo 3). Two $8.5 \mathrm{Fr}$ external drainage catheters (COOK, American) were inserted into the intervertebral disc through the guide wire (Photo 4). After the operation, one external drainage catheter (COOK, American) was used for irrigating saline and

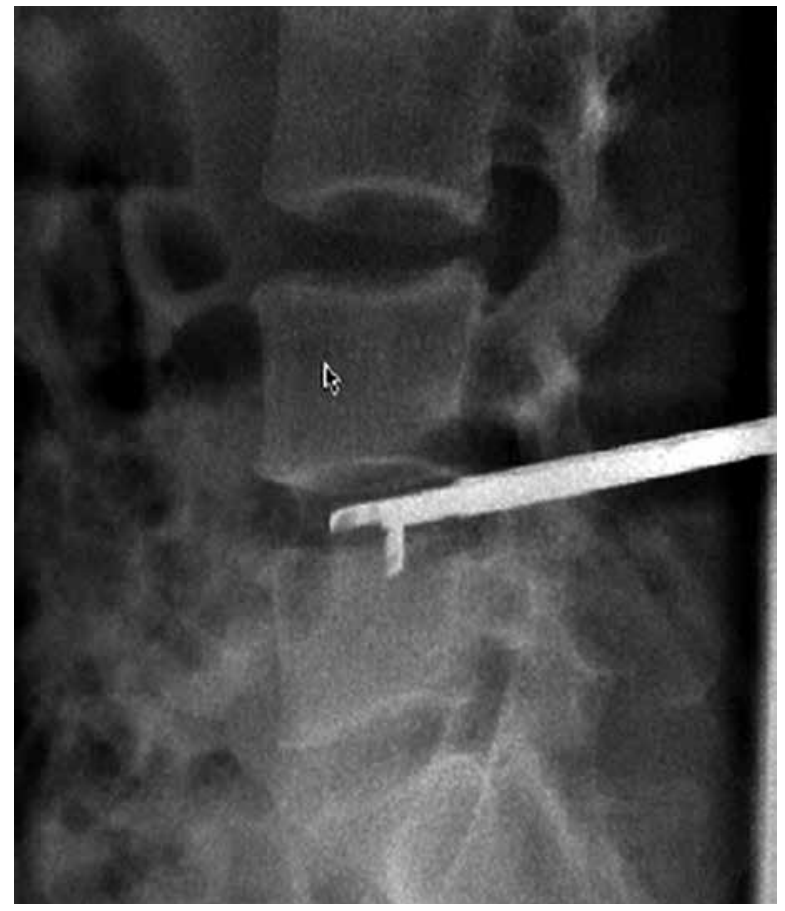

Photo 2. A fluoroscopy shows the nucleus pulposus clamp is inserted into the intervertebral disc through work casing 


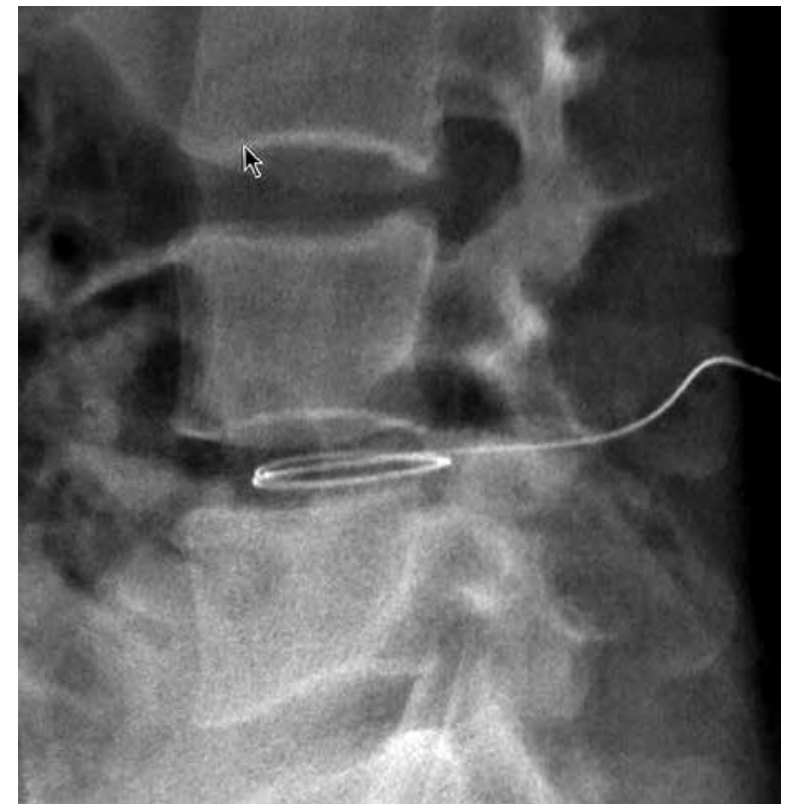

Photo 3. A 0.035 inch guide wire (COOK, American) was inserted through the work cannula

another external drainage catheter (COOK, American) was connected to a drainage pack, which was maintained all the time. The input and output were recorded every day. Meanwhile, systematic antibiotic therapy was also administered. Postoperation CT (Toshiba Aquilion, Japan) and MRI (GE HDxt-3T, GE, American) of vertebrae was examined.

\section{Statistical analysis}

A $p$-value of less than 0.05 was considered to be statistically significant. All statistical analyses were performed using the SPSS package, version 16.0 (SPSS, Chicago, Illinois, USA).

\section{Results}

The basic information of patients is shown in Table I. The ages of patients (male 6 , female 6 ) ranged

Table II. Follow-up result

\begin{tabular}{|lcccc|}
\hline Excellent & Good & Fair & Poor & Efficacy (\%) \\
\hline Postoperation & & & & \\
\hline 1 month & 5 & 6 & 1 & 91.6 \\
\hline 6 months & 7 & 4 & 1 & 91.6 \\
\hline 12 months & 4 & 7 & 1 & 91.6 \\
\hline
\end{tabular}

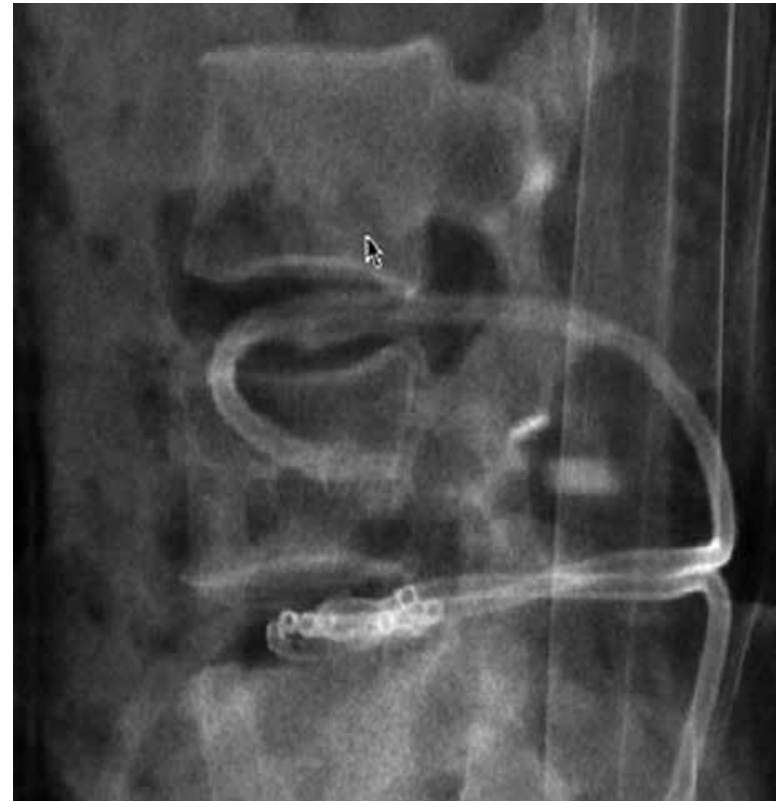

Photo 4. A fluoroscopy shows that two external drainage tubers are inserted into the intervertebral disc

from 47 to 75 years, with a median 58 years. The operation technical success rate was $100 \%$. Preoperation mean VAS score was $8.18 \pm 0.98 ; 5.36 \pm 1.50$ postoperation 1 month; $3.36 \pm 2.24$ postoperation 6 months; $2.77 \pm 0.31$ postoperation 12 months. The comparison of preoperation and postoperation VAS showed a significant difference (all $p<0.05$ ). According to standard Macnab's evaluation, postoperation 12 months, of all 12 cases - excellent 4 cases, good 7 cases, poor 1 case (Table II). No serious complications were recorded. Postoperation efficacy rate was 91.6\%; only 1 case was poor. The postoperation VAS score was dramatically reduced compared with the preoperation score (Figure 1). The comparison of preoperation and postoperation VAS showed a significant difference $(p<0.05)$. Postoperatively the CT and MRI imaging showed that the abscess had disappeared (Photo 5) .

\section{Discussion}

Intervertebral disc infection with epidural abscess is an important problem that often requires aggressive therapy or even surgery. If the epidural abscess is not diagnosed and treated fast, the patient may suffer serious complications such as paraplegia and death [2]. The most frequently affected 


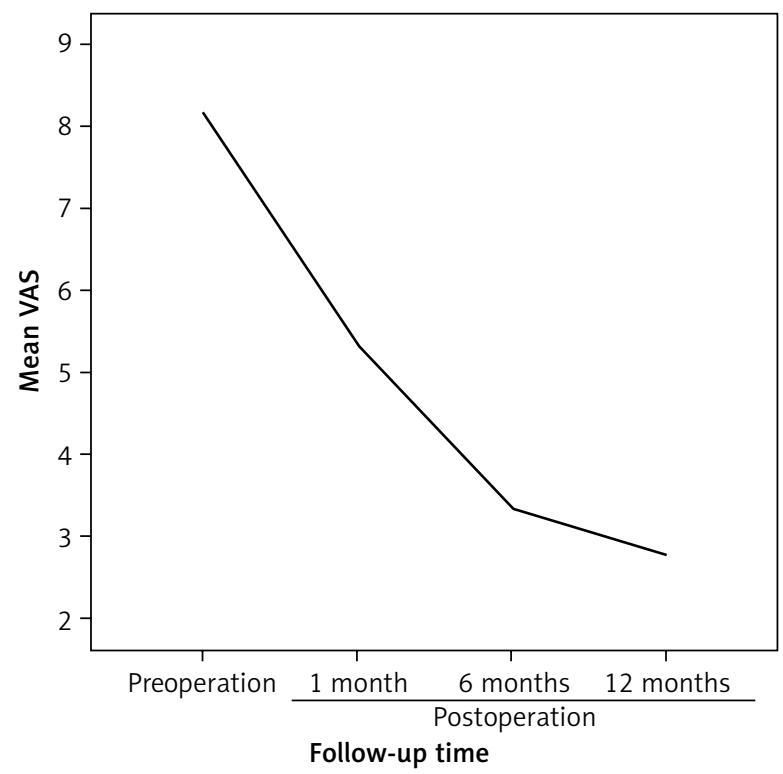

Figure 1 . The variation of VAS of preoperation and postoperation

level was the lumbar level, followed by the thoracic and cervical levels, and lastly, the sacral level. The initial symptoms of intervertebral disc infection with epidural abscess were not specific, and the classical triad of spinal pain, fever, and neurological deficits was present in only $10-15 \%$ of patients at presentation [3]. Measurement of ESR and CPR level, together with radiological diagnostic imaging, was fundamental in the initial assessment and assessment of the patient's response to therapy. ESR was a useful screening parameter, with a 94\% sensitivity [4]. Meanwhile, MRI was the diagnostic test of choice because its sensitivity and specificity are $>90 \%$ [5].

The therapy duration for intervertebral disc infection with epidural abscess depends on the infection-causing microorganism. The recommended duration ranges from 4 weeks to 3 months [6]. Intervertebral disc infection was classified as either pyogenic or granulomatous. For patients with pyogenic infection, Staphylococcus aureus was the most common agent, being isolated in more than $50 \%$ of pyogenic spinal infections [7]. Meanwhile, granulomatous infections are mainly due to brucellosis and tuberculosis. The frequency of intervertebral disc infection in brucellosis ranges from $2 \%$ to $53 \%$ of patients [7]. The incidence rate of tuberculosis intervertebral infection was approximately $50 \%$.

To date, conservative therapy and antibiotic therapy with bracing have been the primary methods for the treatment of intervertebral disc infection. Inter-

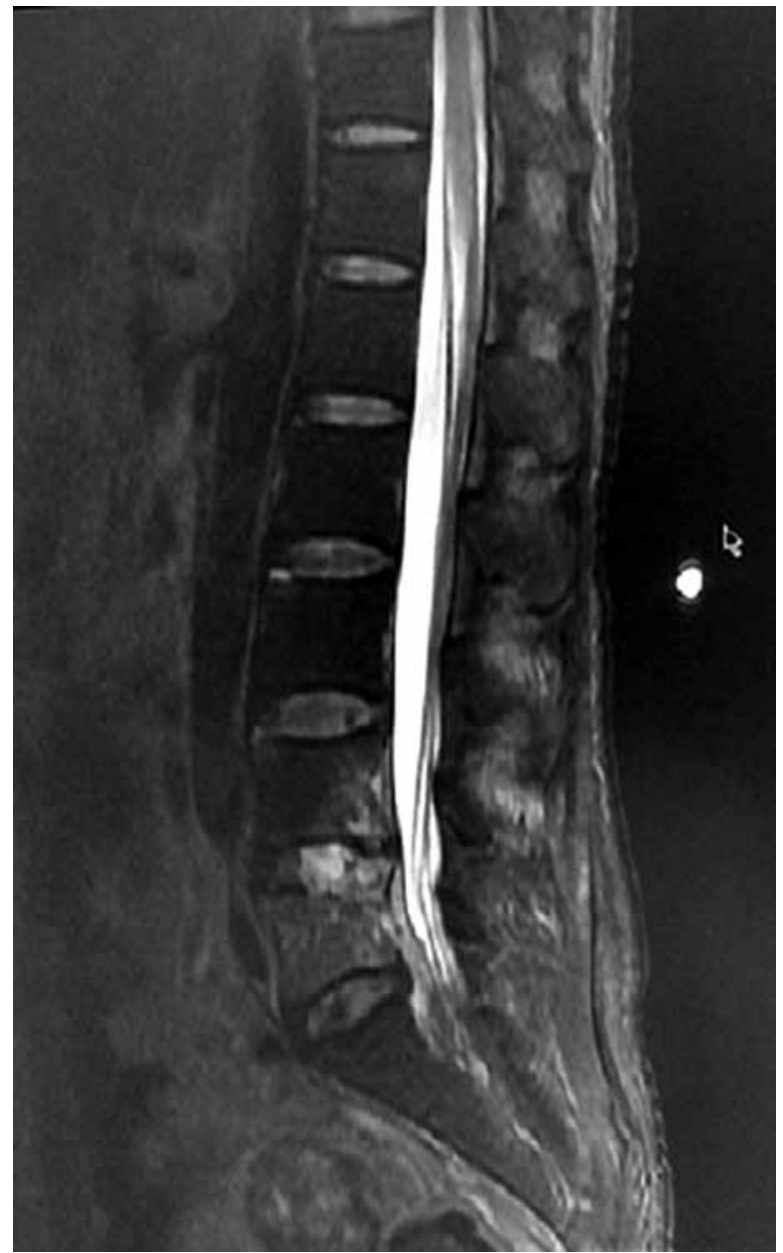

Photo 5. Non-enhanced sagittal T2-weightedMRI reveals that a fusiformis hyperintensity is shrinked and spinal dura sac compressed was dramatically relieved

vertebral disc infection with epidural abscess is an indication for surgery. Once the patient shows any neurologic deficit, surgical decompression should be considered [8]. Although the period of antibiotic therapy for epidural abscess is still being debated, it is preferable to continue treatment for at least 4 weeks, because studies have shown a $25 \%$ rate of relapse in patients who were treated for less than 4 weeks [9-11]. In patients with panspinal epidural abscess, it was impracticable to perform a classic decompressive laminectomy, and in such case less extensive surgery, such as a limited laminotomy with cranial and caudal insertion of epidural catheters for drainage and irrigation, should be considered [12]. For intervertebral disc infection with epidural abscess, surgery was the most frequently reported 
treatment method. Timeothy et al. [13] reported 128 cases of spinal epidural abscess that were medically managed or surgically managed in combination with medical management. They concluded that early surgery could improve neurological outcomes and that medical management failed in approximately $41 \%$ of the patients, requiring surgical decompression. Carefully selected patients can be safely managed with antibiotics alone [4]. Carpenter et al. [14] reported a case of spinal epidural abscess with discitis and vertebral osteomyelitis that was successfully treated with 6-week administration of intravenous antibiotics. Zhang et al. [15] reported a case of cervical epidural abscess with a combination of intravenous vancomycin and imipenem/cilastatin.

In the case of intervertebral disc infection, especially with epidural abscess, performing multilevel laminectomies may predispose the patient to complications such as increased blood loss, cardiac and respiratory difficulties, increased postoperative pain and recovery time, and late kyphotic deformity and instability. At present, instrumentation placement has been accepted during active spinal infection as long as debridement of decreased tissue was complete and an appropriate course of antibiotics was followed [16]. A review of the literature [17] concluded that the infection recurrence rate of spinal instrumentation in the infected spine was $1.74 \%$.

Although intervertebral disc infection with epidural abscess was an indicator of surgery, we used a marrow nucleus rongeur to remove the infected disc material and epidural abscess through the posterior approach and then inserted two external drainage catheters in the intervertebral disc, because the PLD combined with ED was minimally invasive and easy to perform, with little trauma and lower risk for complications compared with surgery. To our knowledge, PLD combined with ED for the treatment of intervertebral disc infections with epidural abscess has rarely been reported. Lyu et al. [18] reported a case of spinal epidural abscess that was treated with percutaneous, computed tomography-guided, needle aspiration and parenteral antibiotic therapy. In this study, we used PLD combined with ED to treat an intervertebral disc infection with epidural abscess and obtained satisfactory results that were similar to those of surgery decompression. Postoperatively the VAS score was dramatically reduced and the pain was relieved. The efficacy was $96.1 \%$; only
1 case result was poor. To our excitement, no serious complications occurred. Compared with surgery, PLD combined with ED does not alter the spinal structure and stability, and it could accelerate the drainage of infectious materials compared with the use of IV antibiotics alone.

Our study had some limitations. Firstly, the number of patients treated by PLD combined with ED was relatively small, which may mask the advantage or disadvantage of the procedure. Secondly, lacking a control group, the clinical efficacy of PLD combined with ED for the treatment of intervertebral disc infection with epidural abscess was not proved to have an advantage over single surgery. So, further research will be carried out in the future.

\section{Conclusions}

PLD combined with ED, together with intravenous antibiotic administration, was demonstrated to be an effective innovative technique for the treatment of intervertebral disc infection with epidural abscess.

\section{Conflict of interest}

The authors declare no conflict of interest.

\section{References}

1. Kaushal M. Result of arthrospine assisted percutaneous technique for lumbar discectomy. Indian J Orthop 2016; 50: 228-33.

2. Murata M, Yoshikawa K, Hiratsuka M, et al. A case of extensive sacral decubitus ulcer complicated by an epidural abscess. Plast Reconstr Surg Glob Open 2016; 4: e790.

3. Davis DP, Wold RM, Patel RJ, et al. The clinical presentation and impact of diagnostic delays on emergency department patients with spinal epidural abscess. J Emerg Med 2004; 26: 285-91.

4. Tompkins M, Panuncialman I, Lucas P, et al. Spinal epidural abscess. J Emerg Med 2010; 39: 384-90.

5. DeSanto J, Ross J. Spine Infection/Inflammation. Radiol Clin N Am 2011; 49: 105-27.

6. Livorsi DJ, Daver NG, Atmar RL, et al. Outcomes of treatment for hematogenous Staphylococcus aureus vertebral osteomyelitis in the MRSA ERA. J Infect 2008; 57: 128-31.

7. Alberto DM, Nicola P, Angela L, et al. Spondylodiscitis: standards of current treatment. Curr Med Res Opin 2012; 28: 689-99.

8. Al-Hourani K, Al-Aref R, Mesfin A. Upper cervical epidural abscess in clinical practice: diagnosis and management. Global Spine J 2016; 6: 383-93.

9. Chan JJ, Oh JJ. A rare case of multiple spinal epidural abscesses and cauda equina syndrome presenting to the emergency department following acupuncture. Int J Emerg Med 2016; 9: 22.

10. Shweikeh F, Saeed K, Bukavina L, et al. An institutional series and contemporary review of bacterial spinal epidural abscess: current status and future directions. Neurosurg Focus 2014; 37: E9. 
11. Suppiah S, Meng Y, Fehlings MG, et al. How best to manage the spinal epidural abscess? A current systematic review. World Neurosurg 2016; 93: 20-8.

12. Hollis PH, Malis LI, Zappulla RA. Neurological deterioration after lumbar puncture below complete spinal subarachnoid block. J Neurosurg 1986; 64: 253-6.

13. Timothy BA, Amit RP, Richard JB, et al. Spinal epidural abscess: risk factors, medical versus surgical management: a retrospective review of 128 cases. Spine J 2013; 13: 45-50.

14. Carpenter W, Afshar N, Mihara K. Spinal epidural abscess with discitis and vertebral osteomyelitis. J Gen Intern Med 2012; 27: 1560 .

15. Zhang JH, Wang ZL, Wan L. Cervical epidural analgesia complicated by epidural abscess: a case report and literature review. Medicine 2017; 96: e7789.

16. Lee MC, Wang MY, Fessler RG, et al. Instrumentation in patients with spinal infection. Neurosurg Focus 2004; 17: E7.

17. Rayes M, Colen CB, Bahgat DA, et al. Safety of instrumentation in patients with spinal infection. J Neurosurg Spine 2010; 12: 647-59.

18. Lyu RK, Chen CJ, Tang LM, Chen ST. Spinal epidural abscess successfully treated with percutaneous, computed tomography-guided, needle aspiration and parenteral antibiotic therapy: case report and review of the literature. Neurosurgery 2002; 51: 509-12.

Received: 12.01 .2020 , accepted: 10.02 .2020 